\title{
How much does skin barrier count for allergic dermatitis improvement?
}

\section{Martins $\mathrm{LM}^{1,2}$, Fialho $\mathrm{LR}^{1}$, Caldeira $\mathrm{J}^{3}$, Silva $\mathrm{N}^{4}$, Antunes $\mathrm{C}^{5}$, Costa $\mathrm{AR}^{5}$, Goicoa $\mathrm{A}^{6}$, Bento $\mathrm{O}^{2,7}$}

\section{Department of Veterinary Medicine, School of Sciences and Technology, University of Évora, Évora, Portugal}

ICAAM, University of Évora, Évora, Portugal

University of Évora Veterinary Hospital, Évora, Portugal

CEVA Saúde Animal, Algés, Portugal

Department of Chemistry, School of Sciences and Technology, and ICT, University of Évora, Évora, Portugal

. Department of Veterinary Clinical Sciences, Hospital Universitario Rof Codina, Veterinary Faculty of Lugo, University of Santiago de Compostela, Lugo, Spain

Department of Animal Sciences, School of Sciences and Technology, University of Évora, Évora, Portugal

\section{Introduction}

\section{Allergic dermatitis is a genetic-based skin condition}

affecting an increasing number of dogs (1). A more

efficient treatment approach is often multimodal, including a special focus on skin barrier condition, from which many pruritic triggers depend as well as common complications (2).

Like in humans, for the development of canine atopic dermatitis ( $C A D)$, defects in the lipid and protein constitution of the skin may contribute to the reduction of the skin barrier function, favoring the in depth penetration of allergens ( 3 ) and stimulating the immune response as an outside/inside - inside/outside paradigm (4).

\section{Results}

Three patients showed evident clinical improvement (No.

$2,3$, and 5$)$ with $(10,19$ and 11 respective decrease in CADESI-4 scores), 1 moderately (No. 4 ) with (3 in CADESI-

4) and 1 (No. 1) did not (1 in CADESI-4)

IDT results did not vary from before to after treatment.

Non-keratinized epidermal layer/stratum corneum thickness ranged respectively $12 / 4(\mathrm{~N})$ to $330 / 300 \mu \mathrm{m}(\mathrm{L})$ before treatment and 10/4 (N) to $120 / 120 \mu \mathrm{m}(\mathrm{L})$ after treatment (Fig.).

Lamina cornea organization tended to increase with no clear change in collagen fibers ( $V G-F i g$.).

Mast cell density and integrity improved in the recovered patients with a mean drop of $15.2 \%$ and an increase of $22.8 \%$, respectively (TB - Fig.).

\section{Aim}

Evaluating the specific role of skin barrier-directed reestablishing treatment in allergic dermatitis clinical improvement

\section{Materials and Methods}

Five dogs with allergic dermatitis were selected from an allergy outpatient consultation following owners' informed consent approved by an ethics committee. Patients were subjected to CADESI-4 evaluation and specific diagnosis for atopy and/or food allergy, including IDT.

Skin biopsies were performed from a non-infected inflamed and adjacent nonaffected area, and conserved in $4 \%$ buffered formaldehyde. Follow up lasted for 2-3 months, applying avoidance measures directed to the implicated allergen sources and treating with a phytosphingosine-containing shampoo/lotion, according to the manufacturer's directions. Reassessment was done each 10-15 days with CADESI-4 and further IDT and skin biopsies performed at the end.

Skin samples were histologically processed including staining with toluidine blue for mastocytes and van Gieson for collagen fibers. Thickness of the non-

keratinized epidermal layer and stratum corneum as well this lamina organization were accessed and scored 1-5. Mast cell density and integrity, and collagen density in the dermal layer were also assessed and scored (\%). Normal

$(\mathrm{N})$ and lesion (L) data, before and after treatment, were compared.

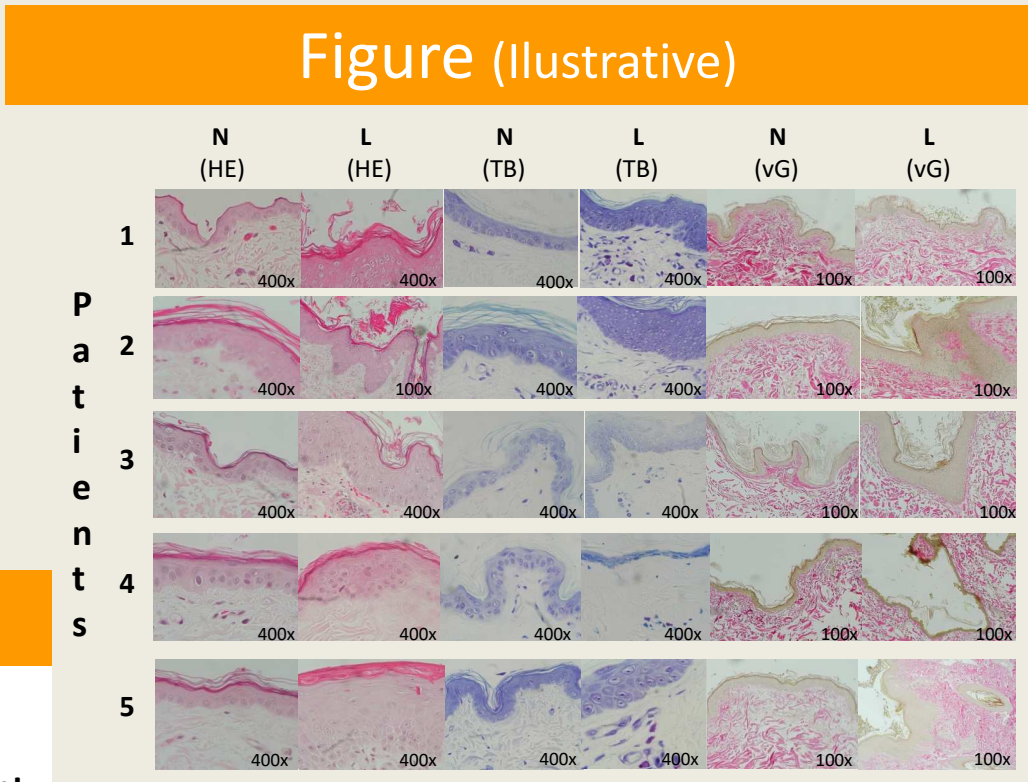

References

1. Halliwell R. Revised nomenclature for veterinary allergy. Vet Immunol Immunopathol 2006;114:207-8

2. Olivry T, DeBoer DJ, Favrot C, Jackson HJ, Mueller RS, Nuttall T, et al. Treatment of canine atopic dermatitis: 2010 clinical practice guidelines from the International Task Force on Canine Atopic Dermatitis. Vet Dermatol 2010;21:233-48

3. Marsella R, Olivry T, Carlotti DN; International Task Force on Canine Atopic Dermatitis. Current evidence of skin barrier dysfunction in human and canine atopic dermatitis. Vet Dermatol 2011; 22:239-48.

4. Marsella R, Samuelson D. Unravelling the skin barrier: a new paradigm for atopic dermatitis and house dust mites. Vet Dermatol 2009;20:533-40. 